\title{
Concepts for a NASA Applied Spaceflight Environments Office
}

\author{
David L. Edwards*a ${ }^{\text {a }}$, Howard D. Burns ${ }^{\mathbf{b}}$, Michael Xapsos ${ }^{\mathrm{c}}, \operatorname{Jim} \operatorname{Spann}^{\mathrm{d}}$ \\ ${ }^{\text {a }}$ National Aeronautics and Space Administration, Marshall Space Flight Center, \\ Mail Code EV44, MSFC, AL, 35812 \\ ${ }^{\mathrm{b}}$ National Aeronautics and Space Administration, Marshall Space Flight Center, \\ Mail Code EM50, MSFC, AL, 35812 \\ ${ }^{\mathrm{c}}$ National Aeronautics and Space Administration, Goddard Space Flight Center, \\ Mail Code 561, Greenbelt, MD 20771 \\ d National Aeronautics and Space Administration, Marshall Space Flight Center, \\ Mail Code VP60, Huntsville AL, 35812
}

\begin{abstract}
The National Aeronautics and Space Administration (NASA) is launching a bold and ambitious new space initiative. A significant part of this new initiative includes exploration of new worlds, the development of more innovative technologies, and expansion our presence in the solar system. A common theme to this initiative is the exploration of space beyond Low Earth Orbit (LEO). As currently organized, NASA does not have an Agency-level office that provides coordination of space environment research and development. This has contributed to the formation of a gap between spaceflight environments knowledge and the application of this knowledge for multi-program use. This paper outlines a concept to establish a NASA-level Applied Spaceflight Environments (ASE) office that will provide coordination and funding for sustained multi-program support in three technical areas that have demonstrated these needs through customer requests. These technical areas are natural environments characterization and modeling, materials and systems analysis and test, and operational space environments modeling and prediction. This paper will establish the need for the ASE, discuss a concept for organizational structure and outline the scope in the three technical areas.
\end{abstract}

\section{INTRODUCTION}

NASA's new initiative to explore new worlds and expand our presence in the solar system presents an achievable and technically challenging objective. Within this initiative are robotic precursor missions to destinations such as Near Earth Objects (NEO), the lunar surface, solar system planets, and the Lagrange points. Additional facets of this new initiative include commercial crew and cargo to transport humans to the International Space Station (ISS), the development of Earth and climate observatories, expanded planetary, astrophysics, and heliophysics science missions. These initiatives and missions will be executed within multiple Directorates and require the expansion of existing programs and establishment of many others. A NASA-level Applied Spaceflight Environments (ASE) function to coordinate applied spaceflight environments knowledge and disseminate that information for Agency, inter governmental, industry, and academia use would provide a cost-effective means to increase our collective knowledge and provide a peer-reviewed suite of tools for engineers and designers to use.

NASA previously supported an Agency-level Space Environments and Effects (SEE) Program that collected, developed, and disseminated the SEE-related technologies required to design, manufacture and operate more reliable and cost-effective spacecraft for the government and commercial sectors. The SEE Program worked in partnership with industry, academia, and other governmental agencies to define the space environment and advocate technology development to accommodate or mitigate the harmful effects on spacecraft. 
The SEE program was terminated in 2005 after a shift in direction by NASA HQ that each program and project would be responsible for the development and implementation of their unique space environment and effects requirements and mitigation. This approach has had marginal success for programs and benefited from the isolated areas of spaceflight environments knowledge scattered throughout the field Centers, other government agencies, industry and academia. The proposed establishment of the ASE office would provide an Agency-level function that would serve to coordinate this knowledge and be able to connect spaceflight designers and engineers with the unique spaceflight environments expertise within NASA, other government agencies, industry, and academia. The products

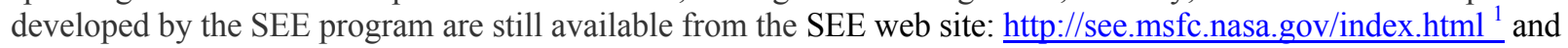
distributed by the Natural Environments Branch at NASA/MSFC. Demand for these products remains high, as will be discussed in the following sections.

The scope of the ASE would address all natural environments that have influence in the design, manufacturing, development, and operation of spaceflight systems. These environments include terrestrial atmospheres, Low Earth Orbit (LEO) to Geosynchronous Earth Orbit (GEO), interplanetary space, planetary atmospheres, and extraterrestrial surfaces. There currently exist several programs focusing on specific components of the space environments, such as meteoroids, orbital debris, and radiation effects on avionics, and the ASE would not include these specific disciplines in its charter. The ASE would establish partnerships with these Programs and help facilitate knowledge flow to the engineering community. The ASE concept would establish an advisory panel with membership consisting of representation from the Office of Chief Engineer (OCE) Space Weather Working Group (SWxWG), the Office of Chief technologist (OCT), Exploration Systems Mission Directorate, and Science Mission Directorate (SMD).

A key feature of this office would be to understand and implement tasks to meet the short-term and long-term needs of the spaceflight community by insuring the products being developed by the ASE are those required by the community. The mechanisms to assess the user product needs are optimized thru the design of the ASE organizational structure. A technical working group structure is envisioned to facilitate stronger technical communication collaboration between the ASE and the spaceflight community using ASE products.

Currently no central location exists for applied spaceflight environments related products, for example requirements documentation, analysis tools, and materials data. Multiple organizations in the agency are performing various tasks pertaining to the space environment but no coordinated effort exists to optimize use of agency resources. NASA has data and associated models on aspects of the space environment, its variability, and effects on materials and components but there is no coordinated function to transition that data in a way that is useful to internal NASA and external users. Programs and projects have been responsible for developing their own specific ASE products. This practice is an inefficient use of NASA resources since it results in duplication of effort in product development and test performance. Establishment of an Agency level ASE will reduce other inefficiencies such as the utilization of tools that are less than optimal, use of outdated tools, and inadequate development time for complex products. These inefficiencies may lead to schedule slips and acceptance of additional risk by the Program.

The ASE will have the experience, expertise, and agency/industry contacts in all aspects of spaceflight environments, effects, and testing to establish and operate an ASE function providing coordinated services to NASA, Government, the commercial aerospace industry, universities, and international partners. These services would include providing a coordinated effort to capture, archive, and share spaceflight environments data (science instrument, materials analysis and test, models, etc) in a way that is useful to the aerospace engineering community.

The ASE would help insure the aerospace user community incorporates an ASE integrated approach to launch vehicle and spacecraft design, development, manufacturing, and operation. The ASE would leverage access to SME's and design specifications to establish standards and guidelines for spacecraft lifecycle relative to environment interaction. 


\section{NATURAL ENVIRONMENTS CHARACTERIZATION AND MODELING}

The technical area of Natural Environments characterization and modeling advocates understanding, characterization and the development of predictive models that accurately define terrestrial, space, planetary surface and planetary atmospheric environments. Existing programs, such as the Meteoroid Environments Office (MEO) are tasked and funded to maintain both sporadic and meteoroid stream models and hence this ASE would not attempt to duplicate existing functions. The ASE would direct inquiries to and establish technical relationships with existing programs.

The SEE Program office was terminated in 2005. Since that time, the Natural Environments Branch at MSFC has continued to distribute the SEE products to the spaceflight community. There are currently environment models available on the SEE website and there have been over 1000 requests for products since 2005 .

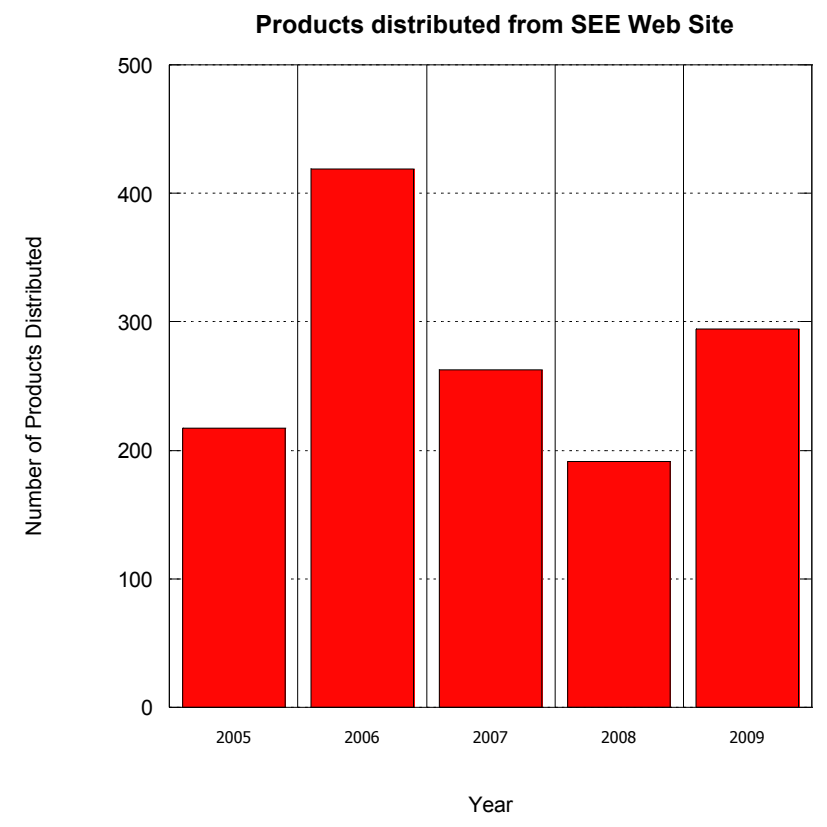

Figure 1. Products requested and distributed from the SEE website since Program termination

These natural environments models are used primarily in the design phase of the Program cycle. These environments give a clear and accurate understanding of the range of environmental parameters in which a vehicle must operate and often drive the design and define the margin of spacecraft performance. Accurate environments models are necessary for mission architecture designers to establish comparable trade studies, and launch vehicle and spacecraft designers to understand the design margin as influenced by natural environments. Many of these products on the SEE website are over 10 years old and in need of updating with state-of-the-art data sets.

Engineering models of the long-term space radiation environment, i.e., climatological models are essential during the mission concept, planning and design phases for risk minimization. During the mission concept period they help select primary technologies. They are used for establishing mission success criteria and architecture trades during the planning phase. They are subsequently used by designers to make trades between risk, cost and performance for components used in microelectronic and photonic subsystems and instrumentation, as well as establishing shielding levels and single event effects mitigation approaches.

Thus, the benefits of these engineering models can be increased use of high performance commercial electronics, lower risk of spacecraft anomalies, and increased payload resources. The models of concern include those of particles trapped in planetary magnetic fields. For the case of Earth orbiting spacecraft this would be primarily protons and electrons in the Van Allen Belts. Also important for risk analysis are solar particle events, which are bursts of radiation emitted from the sun. 
Galactic cosmic rays are relatively low intensity but very high energy particles that originate outside of our solar system. Finally, there is the low energy plasma environment of electrons, protons and other ions which can cause charging of spacecraft materials.

\section{MATERIALS AND SYSTEMS ANALYSIS AND TEST}

The second proposed technical area for the ASE Program/Office would address "Materials and Systems Analysis and Test". There is a demonstrated need in this area considering: a) a customer pool for Space Environmental Effects testing and analysis exists and includes NASA centers, DoD, commercial space, and materials developers; $b$ ) materials test and analysis data exists in many locations but is not readily available to the aerospace community as a compiled database; and, c) no organized approach exists to develop new data to support game changing technology development.

Many space environment test capabilities exist across the agency and most are complementary, in other words there is minimal duplication between sites. These test facilities are utilized by NASA, its contractors, government agencies, industry and academia. Many unique capabilities offer opportunities to evaluate materials and sub- system components performance in simulated flight environments including weather encounter impact during ascent, micrometeoroid /orbital debris impact, atomic oxygen for low earth orbit, ultraviolet radiation, charged particle radiation, plasma/charging, thermal extremes, and vacuum exposure. Test data and information are maintained at the test location and are not readily available to the user community as a whole. This limitation results in duplication of effort and in some cases, testing under conditions that may vary from the actual environment the component will experience due to test system limitations. This approach is not the best use of limited resources and in the latter case, results in a Program possibly accepting additional risk. As new materials and technologies are developed, adequate ground testing to evaluate applications into flight demonstrations or spacecraft is essential to maximize potential for success. Another key component is continued use of flight experiments for environment characterization, material/component evaluation, and operational verification. Flight experiments are essential to insure environmental models are up to date utilizing the latest data. Flight data also helps insure ground based testing is as representative as possible to real space exposure conditions and helps validate ground test protocol.

Materials contamination, although not a natural environment, significantly affects the engineering performance of external spacecraft materials. This ASE concept includes contamination as a constituent of the materials and systems analysis and test technical discipline. There is an existing contamination knowledge base, developed by the SEE program office, that needs up-dating with new materials, materials ground test results from standard outgassing tests, and the development of contamination outgassing and deposition models. These are examples of contamination tasks that can be supported under this technical discipline.

The interaction synergism of the space environment with spacecraft materials can be studied through ground testing but only realistically characterized through flight experiment. These synergistic interactions effect engineering properties of materials and system performance. The ASE function would establish tasks to increase knowledge of the synergistic interactions and further develop empirical models that enable predictive performance of materials and systems operating in specific space environments.

The development of materials engineering property measurement instrumentation is critical to the understanding of environmental effects. Accurate and relevant engineering properties are required to ascertain design and operational margin in addition to the determination of performance lifetimes. Where appropriate, the development of groundbased engineering property measurement instrumentation, and flight experiment environment and material property measurement will be supported by this technical discipline.

Previous SEE program office knowledgebase products were state of the art until 2005. Since then, the products have not been enhanced due to loss of funding. Reduction in available resources and current economic trends increase the need for a collaborative ASE knowledgebase within the aerospace community. The commercial aerospace community has limited, if any, resources to develop and maintain unique test facilities which may be used minimally during the year. 
NASA has a complete set of space simulation test facilities, many of which are unique, with capacity to support the aerospace community as required. The ASE would develop and maintain a list of ground test facilities and points of contact to facilitate communication and cooperation between these test facilities and the user community.

New focus areas defined in the recent proposed FY11 NASA re-direction will result in new technology development which includes materials, component and systems development, as well as test and flight demonstration. Examples of focus areas requiring interaction with the ASE Program might include promotion of Green Technologies which address development of new material formulations and processing techniques utilizing replacement chemicals. These reformulated materials and processes may lead to variations in material properties or performance when exposed to the space environment. Verification that these new materials have no decreased performance in the space environment will be required. The aerospace community should strive to develop, then consistently incorporate ASE guidelines and approaches for design, development, testing and orbital operations. A coordinated and integrated ASE approach will enable successful material advancements leading to game changing technology implementation into NASA programs. New start-up and small commercial space companies in many cases do not have resources to support adequate qualification testing or completely appreciate the complexities associated with spaceflight environments effects on spacecraft and associated materials. Leveraging NASA, NOAA, DoD, university and industry test capabilities and expertise to optimize materials selection, system design, and spacecraft reliability is cost effective. An additional area of interest to the community is establishing ASE guidelines and providing data which will help minimize concerns and reduce risk to NASA funded programs.

Flight experiments are very costly and also critical to accurate understanding of space environment effects on materials. The ASE function will use the approach to leverage existing flight experiment opportunities through NASA (OCT, LWS SET, etc), DoD, and international partners to provide the spaceflight community with spaceflight data.

The challenges of solar system exploration require high performance materials and systems. The ASE would support space material advancement and space system improvements that enable game changing technology implementation during the design, development, testing and orbital operations.

\section{OPERATIONAL SPACE ENVIRONMENTS MODELING AND PREDICTION}

The increased dependence of the Nation, and indeed society worldwide, on space-based communications and navigation requires the ability to manage residual design risk during mission operations. Thus, predictive operational capability of space environmental conditions is needed. This predictive capability currently does not exist in part because it is difficult to develop operational tools for the complex space weather system. In the same way that terrestrial weather prediction is important for many aspects of our security, economy and society, space weather prediction is becoming more and more essential. NASA has the mandate to understand space including the environment in which space-borne assets are immersed. To that end, the Heliophysics Division of the Science Mission Directorate has a dedicated program called Living With a Star (LWS) to investigate those aspects of the Solar-Terrestrial environment that impact life and society. The GSFC Community Coordinated Modeling Center (CCMC) has as its goal to enable, support and perform the research and development for next-generation space science and space weather models. Neither LWS nor CCMC focuses on the transition of space weather research to applications that can be used for operational environments. Recent efforts at GSFC to articulate the requirements for NASA robotic assets begins to address the needs for NASA, but still does not address the national need to transition research to applications. The US Department of Commerce NOAA Space Weather Prediction Center (SWPC) has a mandate to provide space weather information to the nation's government agencies and industry and US Air Force has a similar mandate for DoD. However, NOAA and the USAF rely almost exclusively on NASA for space-based data. NASA has opened a dialog with the largest user of space weather products, the US Army. As the agency moves the human space program beyond LEO, health and safety for human and robotic assets will be a big issue. This is already recognized by the agency and planning is underway to address this need by providing a path for operational products for the human space program. Discussions with NOAA's head of the Space Weather Predictive Center indicate that there is a real need for model improvement, data visualization, and software tools that can be used by the non-specialist. 


\section{ORGANIZATIONAL STRUCTURE CONCEPT}

One organization concept for the ASE function is to establish a main office that functions at the Headquarters level. This office must have access to and authority to operate across Field Center boundaries. The ASE office staff would consist of one manager, one chief scientist, three technical subject matter experts and a management support assistant. Sufficient resources must be distributed to the participating Field Centers to provide coverage and secure participation in ASE operations.

The technical structure will be developed to support three technical disciplines; Natural Environments Characterization and Modeling, Materials and Systems Analysis and Test, and Operational Space Environments. Each technical discipline would consist of specific technical working groups, as define in figure 2.
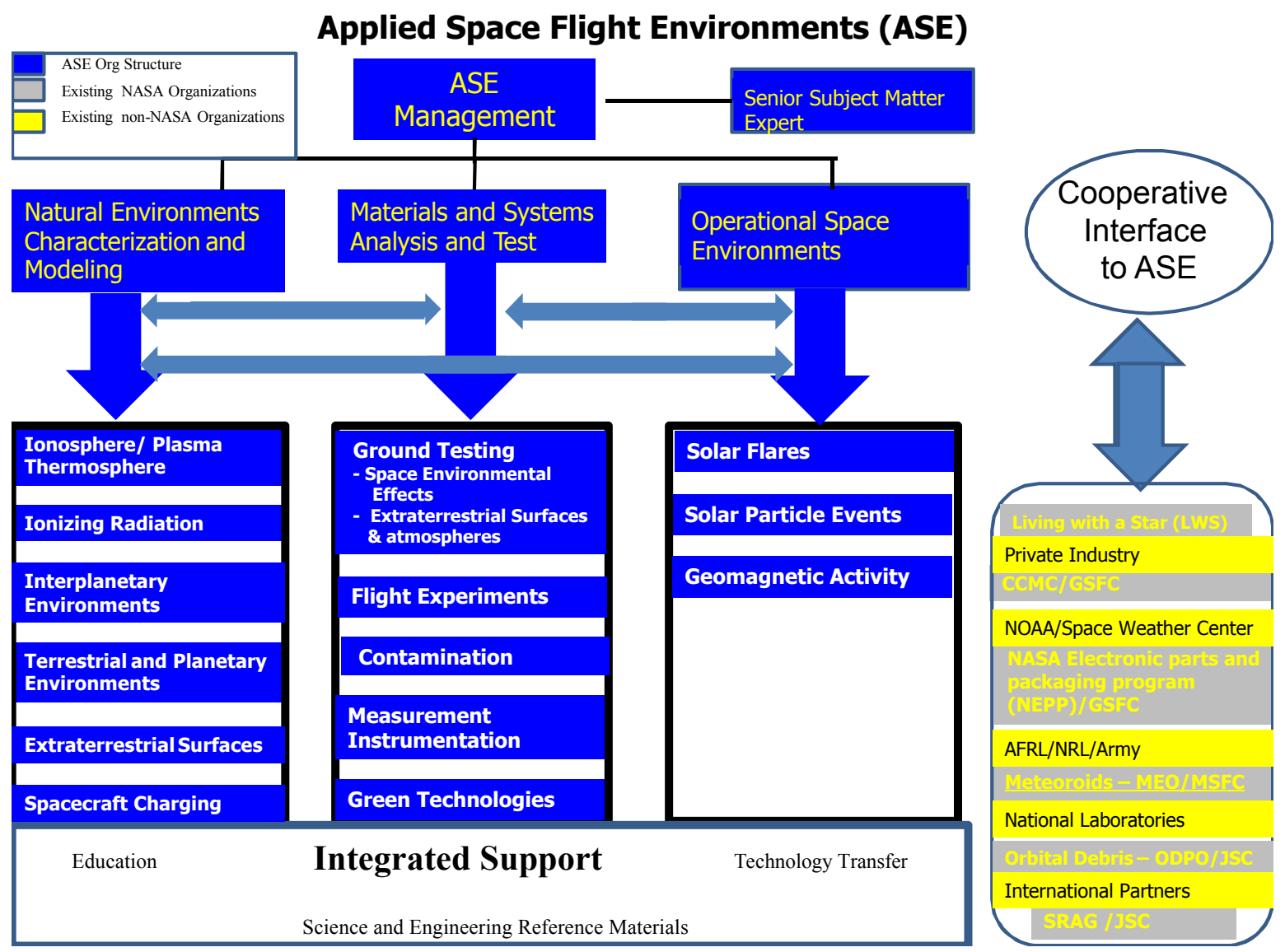

Figure 2. Concept Organizational Structure for the ASE. Each technical discipline will have specific working groups as defined.

These working groups will consist of members from NASA field Centers, other governmental agencies, Industry, academia, and where appropriate International partners. The working group structure will be the primary interface to the product user community and thus provide the critical feedback to decisions on product development for the coming year. Education, technology transfer, and the development of science and engineering reference materials is deemed to be a necessary and intended spin-off from the products developed by this ASE function. 


\section{SUMMARY AND DISCUSSION}

The concept for an Applied Spaceflight Environments (ASE) function was described. This function would be organizationally positioned at the Headquarters level to provide equal access to all NASA Field Centers. The ASE function would develop products identified and prioritized by the user community and proactively develop long lead-time products to avoid the approach of developing critical tools only when faced with requirement deadlines. The ASE function will serve to gather knowledge from the spacecraft community and develop, archive, and disseminate products to the spaceflight community. The ASE will provide a forum for spaceflight environment subject matter experts to collaborate and advance the state of knowledge. There are numerous users who continue to request and receive products from the now terminated SEE program web site. Most products were developed ten years ago or longer. This continued demand for SEE products demonstrates a need for an organization that funds product development and disseminates these products to the spaceflight community.

The current status of this concept is in the socialization and refinement stage. Spaceflight environment experts from across NASA have been introduced to this concept and had an opportunity to review and comment to help shape and strengthen this idea. Discussions are being held with Mission Directorates at NASA HQ and key personnel in the Office of the Chief Technologist (OCT) as well as the Office of the Chief Engineer (OCE). At the time of this writing, there is not an organization that has demonstrated support to sponsor this ASE function.

\section{ACKNOWLEDGEMENT}

The authors would like to thank and acknowledge the Space Environments and space materials organizations at MSFC, GRC, JPL, and GSFC. Their input and comment to the development of this concept has been invaluable. The authors would like to specifically acknowledge the following individuals for their support, guidance, and recommendations as this concept for the ASE matured: Dr. Hank Garrett and Dr. Paul Willis at JPL, Dr Viet Nguyen and Dr. Mike Piszczor at GRC, Dr. Rob Suggs, Mr. Todd May, Ms. Bonnie James, Ms. Mary Nehls, and Dr. Andrew Keys at MSFC, Dr. John Lyver and Dr. Dana Brewer at HQ.

\section{REFERENCES}

1. http://see.msfc.nasa.gov/index.html, Space Environments and Effects Program Office website. 


\section{Concepts for a NASA Applied Spaceflight Environments (ASE) Office}

\section{David L. Edwards, Howard D. Burns, Michael Xapsos, Jim Spann}

June 30, 2010 


\section{Problem Statement:}

A gap exists between spaceflight environments knowledge and the application of this knowledge for multi-program, cross cutting use.

- Presently, each program/project must fund, establish, and develop their individual spaceflight environments products with the potential consequences of:

- $\quad$ Duplication of effort

- $\quad$ Over/under engineering

- $\quad$ Using inappropriate critical information

- $\quad$ Acceptance of risk

\section{Solution Statement:}

To resolve this problem, establish a NASA organization that will provide coordination and funding for sustained multi-Program support in three technical areas that have a demonstrated need through customer pull. These technical areas are:

Natural Environments Characterization and Modeling

Materials and Systems Analysis and Test

Operational Space Environments Modeling and Prediction 


\section{Natural Environments Characterization and Modeling}

\section{Demonstrated need:}

- MSFC/EV44 maintains the Space Environments \& Effects (SEE) Program web site and continues to distribute SEE products to the NASA, US, and the international aerospace community even though the SEE Program was terminated in 2005.

- No central NASA Organization exists to develop these unique SEE products.

- No funding is available to improve the SEE products.

- Over 1000 requests for products have been received and products were distributed in the last 5 years.

- NASA, Government, and Aerospace Industry Programs use these SEE products.

- Most SEE products are approaching 10 years without update.

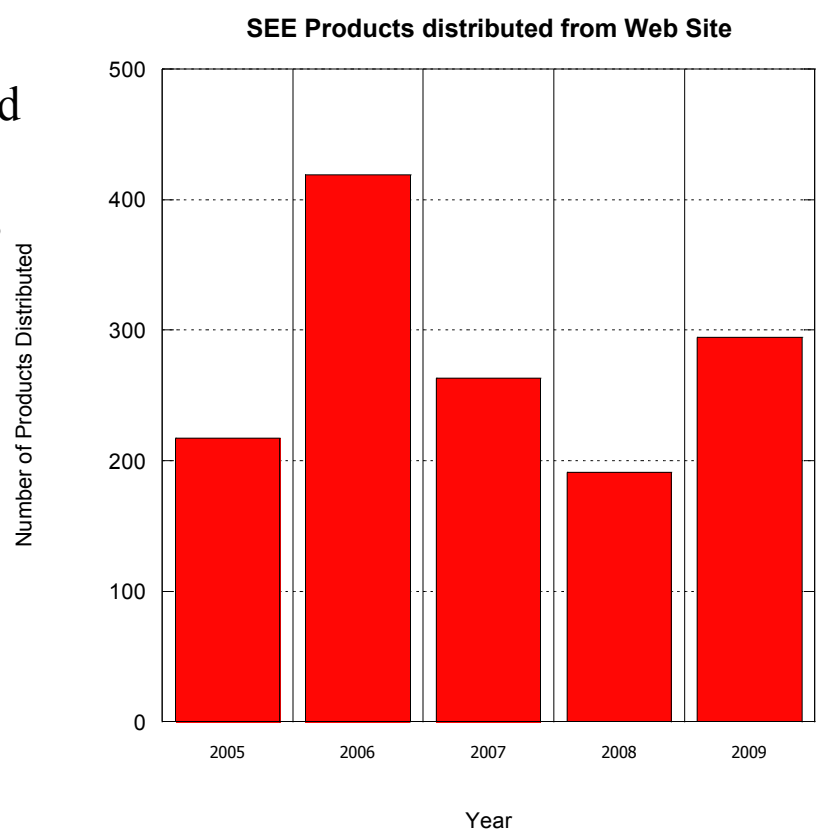

http://see.msfc.nasa.gov/index.html 


\section{Materials and Systems Analysis and Test}

\section{Demonstrated need:}

- Customer pool for Space Environmental Effects testing and analysis - includes NASA centers, DoD, commercial space, and materials developers

- Materials test and analysis data exists in many locations but is not readily available to the aerospace community as a compiled database

- No organized approach exists to develop new data for game changing technology development

- Comm-NGC

Space Environmental Effects Testing at MSFC/EM50 2008-2010

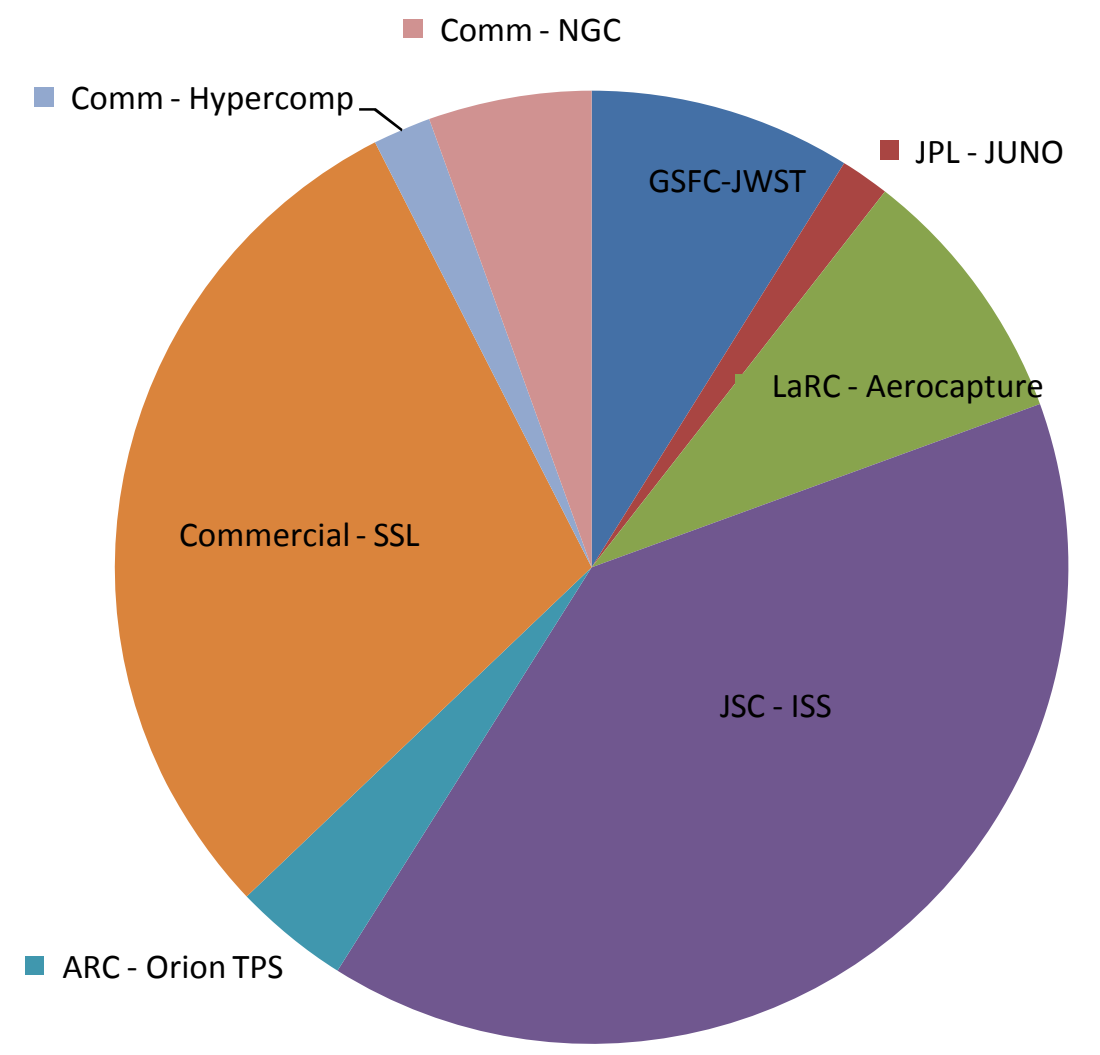




\section{Operational Space Environments Prediction}

\section{Example Demonstrated need:}

- For space-weather forecasting NOAA and others currently use a version of the McIntosh classification scheme to predict the occurrence rate of $\mathrm{X}$ and $\mathrm{M}$-class solar flares.

- These are the largest and most dangerous flares produced by the Sun and their affects on spaceflight systems must be accounted for in flight operations.

- The graph compares the performance of the McIntosh prediction scheme against the MSFC All-Clear model.

- The existing prediction model seriously under estimates periods of high flare occurrence, which the new MSFC model provides more than a $200 \%$ improvement.

- The MSFC model is now in certification testing by NASA/JSC

\section{Scope of need examples:}

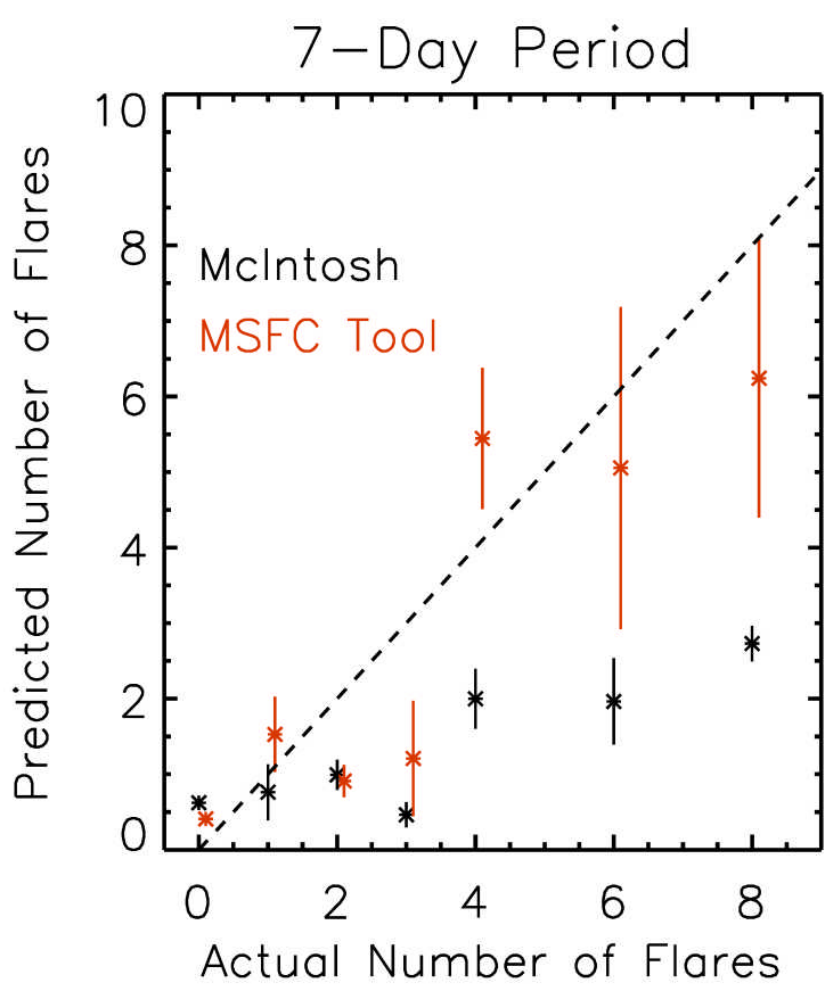

- $\quad$ Space weather launch support for NASA DART mission

- Environment assessments for anomaly investigations: ISS charging environments, CALIPSO, Pluto New Horizons, and Chandra radiation environments working group

- Spacecraft operations, especially high voltage power supplies that can be compromised by penetrating radiation

- Aircraft high latitude flight plans are changed when radiation exposure hazard is enhanced 


\section{Why the ASE is Needed}

- Many current tools are 10 years out of date

- Significant amounts of Science data are available; not in form to use for engineering design

- Promotion of Green Technologies related to ASE

- A Coordinated, integrated approach to ASE would enable material advancement leading to game changing technology implementation

- Help insure Aerospace community consistently incorporates ASE approaches for design, development, testing and orbital operations

- New start-up commercial space companies do not adequately understand spaceflight environments effects on spacecraft and associated materials concerns

- Provide flight experiment opportunities for industry, universities, NASA, and DoD

- Inadequate set of ASE related requirements and implementation approaches

- NASA has data and associated models on space environment, its variability, and effects on materials and components but there is no coordinated program to transition that data in a way that is useful to internal and external users

- Multiple organizations in the agency are doing various parts of ASE but no coordinated effort exists to optimize use of agency resource

-Many users who need this information don't know where to go, or how to use the data and models once they find it 


\section{The ASE Organization will fulfill the needs of multiple users (NASA, Other Government Agencies, US Aerospace Industry, Universities, and International Partners) by :}

- Proactively developing long-lead time tools, updating existing tools and data sets, and conducting materials/subsystem evaluation testing needed by NASA and the spacecraft community.

- Collecting knowledge from the aerospace and space users communities through actively interfacing with known users and with working group/survey-type forums to ascertain technology needs.

- Offering flight experiment opportunities for the ASE community.

- Providing a coordinated effort capture, archive, and share spaceflight environments data (science instrument, materials analysis and test, models, etc) in a way that is useful to the aerospace engineering community.

- Helping insure the aerospace and space user communities incorporates an ASE Program integrated approach to launch vehicle and spacecraft design, development, manufacturing, and operation.

- Enabling material advancement and space system improvements leading to game changing technology implementation during design, development, testing and orbital operations.

- Providing a forum for Subject Matter Experts (SME's) to advance the ASE knowledgebase.

- Leveraging NASA, NOAA, DoD, university and industry test capabilities and expertise to optimize materials selection, system design and spacecraft reliability.

- Leveraging experience with environments, effects, and current Earth Science Application programs (SERVIR, SPoRT) to transition from data/models to useful tools that meet customer needs. 


\section{The ASE concept has been shared with other field centers and agencies with positive response}

\begin{tabular}{|l|l|}
\hline Institution & Contact \\
\hline HQ & $\begin{array}{l}\text { Dick Fisher, Vicki Elsberned, John } \\
\text { Lyver }\end{array}$ \\
\hline MSFC & $\begin{array}{l}\text { Todd May, Andrew Keys, Bonnie } \\
\text { James (HQ/OCT) }\end{array}$ \\
\hline GSFC & Michael Hesse, Jim Slavin \\
\hline GRC & Viet Nguyen, Mike Piszczor \\
\hline JPL & Hank Garrett, Paul Willis \\
\hline NOAA & Tom Bogdan, Terry Onsager, Bill \\
\hline US Army SMDC & Lapenta, Doug Biesecker \\
\hline ONR & Larry Burger \\
\hline
\end{tabular}




\section{First Year Approach}

- ASE office - 6 EP (manager, 1 program Scientist, 3 technical assistants, 1 scheduler/coordinator)

- Technical support - 8 EP spread across participating Centers

- Total estimated budget approximately :

- 14 EPs

- $\$ 5 \mathrm{M}$ in Procurement for task-based product development

- $\$ 125 \mathrm{~K}$ in operating budget (Includes travel for $14 \mathrm{EP}$ )

- Seek commitment from all participants across ASE community

- Establish Space Act Agreements, Task Agreements, etc between parties

- Identify SMEs, working groups, and ASE capabilities across aerospace community

- Query to identify ASE technical area needs

- Determine resource (funding, personnel, etc) requirements and develop 3 year business plan

- Establish schedule for holding conference/workshop/working group meetings to define way forward (priorities, ...)

- Hold ASE kick-Off workshop

- Leverage existing capacity where available, for example:

- Meteoroid Environments Office (MEO)

- Orbital Debris Program Office (ODPO)

- NASA Electronic Parts and Packaging (NEPP)

- Community Coordinated Modeling Center (CCMC)

- Space Weather Working Group 


\section{Follow-on Approach / Plan}

- Maintain small Program Office staff

- Query Aerospace community/Users regularly to ascertain technology needs

- Fund products with Multiple program applications;

- Single application products require Program/project funding

- Disseminate Products to the public

- Leverage existing capacity where available, for example:

- Meteoroid Environments Office (MEO)

- Orbital Debris Program Office (ODPO)

- NASA Electronic Parts and Packaging (NEPP)

- Community Coordinated Modeling Center (CCMC)

- Space Weather Working Group

- Living With a Star (LWS) proposal winners

- Maintain a central authority on ASE thru:

- Working group participation

- Subject Matter Experts (SME's) throughout the world

- Knowledge of specialized facilities to assess effects of Spaceflight Environments

- Program will sponsor both competitive and direct funded tasks

- Competition open to NASA, Other Government Agencies, US Aerospace Industry,

Universities, and if applicable - International Partners

- Coordinate with SMD LWS Program

- Propose the Agency fund the Program with sponsorship from multiple Mission Directorates, other Agencies (NOAA), and DOD

- Annual budget - varying depending on tasks, products, flight experiments, etc desired by the ASE community

- Estimated at $\$ 4-5 \mathrm{M}$ annually 
Back up Slides 
Applied Space Flight Environments (ASE)

ASE Org Structure

Existing NASA Organizations

Existing non-NASA Organizations
Natural Environments Characterization and Modeling

\begin{tabular}{l}
\hline \\
\hline Ionosphere/ Plasma \\
Thermosphere \\
\hline Ionizing Radiation \\
\hline Interplanetary \\
Environments \\
\hline Terrestrial and Planetary \\
Environments \\
\hline
\end{tabular}

Education

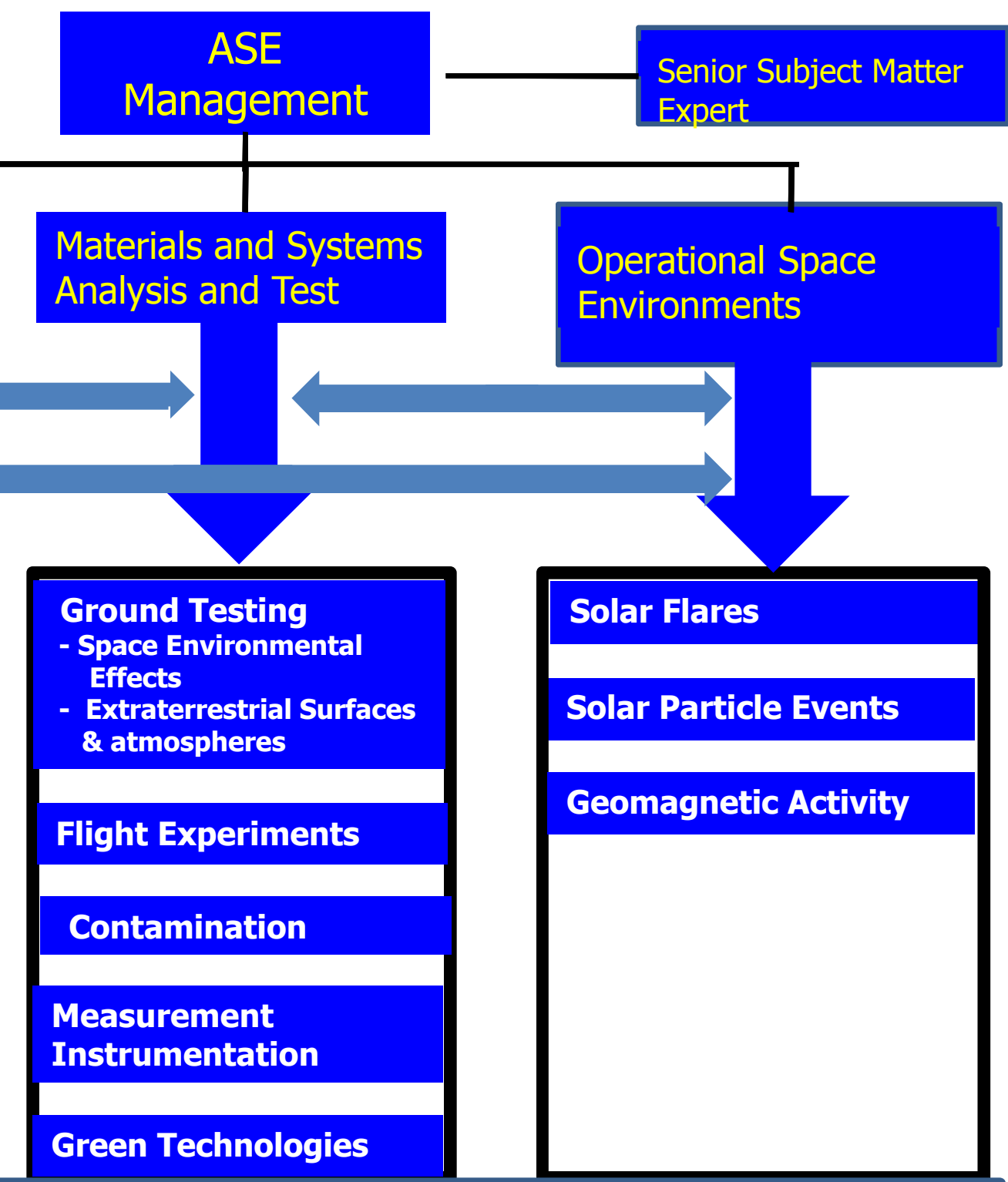

\section{Integrated Support}

Technology Transfer
Cooperative Interface to ASE

Living with a Star (LWS)

Private Industry

CCMC/GSFC

NOAA/Space Weather Center

NASA Electronic parts and packaging program (NEPP)/GSFC

AFRL/NRL/Army

Meteoroids - MEO/MSFC

National Laboratories

Orbital Debris - ODPO/JSC

International Partners

SRAG /JSC 


\section{First Year Acquisition Options}

- Host Applied Space Flight Environments Workshop

- Identify flight experiment opportunities and buy in participation to support early flight demonstrations

- Task the CCMC for model/tool development

- Task with SAIC to upgrade/improve NASCAP-2K

- Participate in Near Earth Object Characterization

- Initiate task to Characterize NEO environments for robotic and/or manned missions

- Initiate wind pairs database generation at KSC and Wallops

- Partner with ROSES NRA to expand proposal opportunities in Spaceflight Environments

- Initiate improvements to the following Models:

- Mars GRAM, Electric Propulsion Interactions Code( EPIC),

Emission of Solar Protons (ESP)

- Develop a deep dielectric charging model

- Develop guidelines on Integrated approach to spaceflight environments

- CREME MC

- All Clear Forecast Tool 